\title{
Bridging the mental-physical divide in health care
}

\author{
Mark Sinyor MSc MD, Benjamin I. Goldstein MD PhD, Ayal Schaffer MD
}

- Cite as: CMAJ 2019 July 2;191:E722-3. doi: 10.1503/cmaj.190709

See related article at www.cmaj.ca/lookup/doi/10.1503/cmaj.181712

I

$\mathrm{t}$ is well established that mental disorders account for a substantial proportion of years lived with disability, with depressive disorders alone accounting for 50 million years lived with disability worldwide in 2015. ${ }^{1}$ Patients with comorbid medical and psychiatric illness are known to have increased health care costs, length of stay in hospital and rates of hospital readmission compared with those without medical comorbidity. ${ }^{2}$ How mental and physical comorbidity influence people's health trajectories has crucial implications for the way we fund and organize health care, and even for our understanding of the illnesses themselves. However, a nuanced understanding of the relationship between psychiatric and medical conditions remains elusive.

In a linked study, Gaulin and colleagues used logistic regression modelling to show synergistic effects of comorbid mental and physical illnesses on frequent visits to the emergency department (defined as $\geq 3 / \mathrm{yr}$ ) across the province of Quebec. ${ }^{3}$ That is, the combination of the 2 types of disorders was associated with more visits than would be expected if one assumed a simple additive effect. Specifically, the researchers found that synergy between mental and physical disorders accounted for $13 \%-24 \%$ of emergency department visits in those with mental disorders. It also accounted for $5.8 \%$ of all emergency department visits across the province (24388 additional visits over 14 months). This would equate to more than $\$ 15$ million in yearly excess health spending on emergency department visits alone, excluding physician billings. ${ }^{4}$

Gaulin and colleagues further found that the synergistic effect was more pronounced in those with serious mental disorders (psychotic or bipolar disorders). It may be that the magnitude of effect is underestimated, as the authors had to rely on diagnosed medical and psychiatric comorbidities, and disparities in diagnosis and treatment of physical disorders are known to exist among individuals with serious mental disorders. ${ }^{5}$

The linked study used rigorous methodology to show and quantify what should be intuitive to those of us who routinely treat patients with comorbid psychiatric and medical conditions. It contributes to a growing understanding of the interrela-

\section{KEY POINTS}

- New research adds to growing scientific knowledge showing the important influence of physical comorbidity on use of health care resources in mental disorders.

- This evidence has important implications for how we conceptualize mental illness, and how we organize and fund health care.

- Correcting the inaccurate historical schism between physical and mental health requires us to rethink health care strategy, organization and funding.

tionship between mental and physical illness and suggests the need for a parallel synergy in the treatment strategy of these conditions, which is already beginning to occur. The emergence of the anesthetic ketamine as a treatment for depression and suicidal ideation has led to greater collaboration between psychiatrists and anesthetists in hospital settings. ${ }^{6}$ In 2015, one of us (B.G.) became the first psychiatrist to lead a scientific statement by the American Heart Association that highlighted the link between mood disorders and cardiovascular disease. ${ }^{7}$ At a system level, the Medical Psychiatry Alliance in Ontario is Canada's first provincially funded collaboration to improve knowledge, expertise and treatment models for comorbid mental and physical illnesses, as well as quality of life, in sufferers. ${ }^{8}$

Just as we understand the heart and lungs to be separate but interdependent organ systems, the field of medicine is increasingly recognizing brain and body as a single system. Correcting the inaccurate historical schism between the two requires us to rethink health care strategy, organization and funding. Research regarding treatment outcomes for comorbid mental and substance use disorders, for example, has repeatedly shown that integrated treatment outperforms parallel or sequential treatment strategies. ${ }^{9}$ An analogous approach may yield similar benefits for mental and physical comorbidity. That would require continued efforts to reduce the stigma of mental disorders that remains in medicine and to shift from viewing 
these disorders as an impediment to treatment of physical disorders, toward a justification for more intensive patient care efforts. It would also require us to more clearly position traditional primary and secondary prevention efforts, such as healthy diet and exercise counselling, as being equally important for mental and physical health.

Such a change, long overdue, might also benefit from a reconsideration of how we position mental disorders within medicine overall. Currently, a myocardial infarction and a tibial fracture are both considered "physical" health problems, and major depression a "mental" health problem. Yet there appears to be a relationship between the pathophysiology, specifically endocrine changes and inflammatory and immunological activation, that underlie both cardiovascular disease and depression, ${ }^{10}$ and antidepressants may even lower mortality for patients after myocardial infarction. ${ }^{11}$ These findings underscore the need for a reorganization of our conceptual frameworks of illness and greater integration between our approaches to what were previously considered disparate conditions.

The Canadian Mental Health Association has called for a national Mental Health Parity Act, like the one already in existence in the United States, to create greater parity in mental health funding and access to mental health care. ${ }^{12}$ Although such a step is an important one for the future of Canadian health care, we must carefully consider the implications of studies, like the one linked here, for that effort. There will certainly remain a role for independent mental health providers and facilities in the near to mid-term, but the close interplay between mental and physical disorders strongly suggests that health care should ideally be provided via a collaborative approach in centres with expertise in both types of conditions. Although standalone facilities may exist, we generally do not have gastroenterology hospitals or endocrinology hospitals, owing to an acknowledgement that many other areas of medicine coexist and affect conditions in these areas. The evidence suggests that treatment of mental disorders likewise must be integrated within hospitals and the greater health system.

We work in one of Canada's largest teaching hospitals and, like many of our psychiatry colleagues working in hospitals around the country, routinely collaborate both clinically and in research with internists, anesthetists and colleagues from other medical and surgical specialties. These interactions yield reciprocal benefits that clearly improve the care, health and well-being of our patients. The study by Gaulin and colleagues is an important reminder of why we organize our hospital and our practices in this way, and of the need for this approach to become the standard of care across Canada.

\section{References}

1. Depression and other common mental disorders: global health estimates. Geneva: World Health Organization; 2017.

2. Jansen L, van Schijndel M, van Waarde J, et al. Health-economic outcomes in hospital patients with medical-psychiatric comorbidity: a systematic review and meta-analysis. PLoS One 2018;13:e0194029.

3. Gaulin M, Simard M, Candas B, et al. Combined impacts of multimorbidity and mental disorders on frequent emergency department visits: a retrospective cohort study in Quebec, Canada. CMAJ 2019;191:E724-32.

4. Sinyor M, Williams M, Belo S, et al. Ketamine augmentation for major depressive disorder and suicidal ideation: preliminary experience in an inpatient psychiatry setting. J Affect Disord 2018;241:103-9.

5. Ramasubbu R, Beaulieu S, Taylor VH, et. al; Canadian Network for Mood and Anxiety Treatments (CANMAT) Task Force. The CANMAT task force recommendations for the management of patients with mood disorders and comorbid medical conditions: diagnostic, assessment, and treatment principles. Ann Clin Psychiatry 2012;24:82-90.

6. Patient accounts. Montréal: McGill University Health Centre; 2019. Available: https://muhc.ca/homepage/patientaccounts (accessed 2019 June 6).

7. Goldstein BI, Carnethon MR, Matthews KA, et al.; American Heart Association Atherosclerosis; Hypertension and Obesity in Youth Committee of the Council on Cardiovascular Disease in the Young. Major depressive disorder and bipolar disorder predispose youth to accelerated atherosclerosis and early cardiovascular disease: a scientific statement from the American Heart Association. Circulation 2015;132:965-86.

8. Medical Psychiatry Alliance [home page]. Available: www.medpsychalliance. ca/ (accessed 2019 June 6).

9. Kelly TM, Daley DC. Integrated treatment of substance use and psychiatric disorders. Soc Work Public Health 2013;28:388-406.

10. Vaccarino V, Badimon L, Bremner JD, et al.; ESC Scientific Document Group Reviewers. Depression and coronary heart disease: 2018 ESC position paper of the working group of coronary pathophysiology and microcirculation developed under the auspices of the ESC Committee for Practice Guidelines. Eur Heart J 2019 Jan. 28. [Epub ahead of print]. doi: 10.1093/eurheartj/ehy913.

11. Taylor CB, Youngblood ME, Catellier D, et al.; ENRICHD Investigators. Effects of antidepressant medication on morbidity and mortality in depressed patients after myocardial infarction. Arch Gen Psychiatry 2005;62:792-8.

12. Mental health in the balance: ending the health care disparity in Canada. Toronto: Canadian Mental Health Association; 2018. Available: https://cmha.ca/wp-content/ uploads/2018/09/CMHA-Parity-Paper-Full-Report-EN.pdf (accessed 2019 May 28).

\section{Competing interests: None declared.}

This article was solicited and has not been peer reviewed.

Affiliations: Departments of Psychiatry (Sinyor, Goldstein, Schaffer) and Pharmacology (Goldstein), Sunnybrook Health Sciences Centre; Department of Psychiatry (Sinyor, Schaffer), University of Toronto; Centre for Youth Bipolar Disorder (Goldstein), Department of Psychiatry, Sunnybrook Health Sciences Centre, Toronto, Ont.

Contributors: All authors contributed to the design of the manuscript, revised it critically for important intellectual content, gave final approval of the version to be published and agreed to be accountable for all aspects of the work.
Competing interests: Mark Sinyor reports that he has received grant support from the American Foundation for Suicide Prevention, the Ontario Ministry of Research and Innovation, the Innovation Fund of the Alternative Funding Plan from the Academic Health Sciences Centres of Ontario, the University of Toronto Department of Psychiatry Excellence Fund, and Academic Scholar Awards from the Departments of Psychiatry at the University of Toronto and Sunnybrook Health Sciences Centre.

Correspondence to: Mark Sinyor, mark.sinyor@sunnybrook.ca 